FERMILAB-TM-2052

\title{
Beam Stability Issues of 3 TeV Low-Field Collider
}

\author{
King-Yuen Ng \\ Fermi National Accelerator Laboratory \\ P.O. Box 500, Batavia, Illinois 60510
}

August 1998 


\section{Disclaimer}

This report was prepared as an account of work sponsored by an agency of the United States Government. Neither the United States Government nor any agency thereof, nor any of their employees, makes any warranty, expressed or implied, or assumes any legal liability or responsibility for the accuracy, completeness, or usefulness of any information, apparatus, product, or process disclosed, or represents that its use would not infringe privately owned rights. Reference herein to any specific commercial product, process, or service by trade name, trademark, manufacturer, or otherwise, does not necessarily constitute or imply its endorsement, recommendation, or favoring by the United States Government or any agency thereof. The views and opinions of authors expressed herein do not necessarily state or reflect those of the United States Government or any agency thereof.

\section{Distribution}

Approved for public release; further dissemination unlimited. 
TM-2052

\title{
BEAM STABILITY ISSUES OF 3 TeV LOW-FIELD COLLIDER
}

\author{
King-Yuen Ng \\ Fermi National Accelerator Laboratory, ${ }^{*}$ P.O. Box 500, Batavia, IL 60510
}

(August, 1997)

\section{INTRODUCTION}

We analyze the stability issues of the $3 \mathrm{TeV}$ low-field collider. Some relevant properties of the collider are listed in Table I. In the table, the rms bunch length of $\sigma_{\ell}=0.50 \mathrm{~m}$ and bunch area of $A=1.50 \mathrm{eV}$-s at injection are extraction values from the Main Injector. At extraction of this $3 \mathrm{TeV}$ ring, we assume the bunch area to be the same, but the rf voltage has been cranked up to $V_{\mathrm{rf}}=4.00 \mathrm{MV}$.

\section{MICROWAVE INSTABILITIES}

Both longitudinal and transverse microwave instabilities have growth rates much faster than a synchrotron period. They are driven by broad-band impedances centered at frequency $f_{r}$, corresponding to a wavelength less than the length of the bunch. Therefore, we take $f_{r} \gtrsim \sigma_{\tau}^{-1}$, where $\sigma_{\tau}$ is the rms length of the bunch. The limit for longitudinal microwave stability is

$$
\left|\frac{Z_{0}^{\|}}{n}\right|=\frac{2 \pi E|\eta| \sigma_{E}^{2}}{e I_{\mathrm{pk}}},
$$

which equals 7.12 and $1.01 \mathrm{Ohms}$ at injection and extraction. In the above, $E$ is the total energy and $\sigma_{E}$ the fractional energy spread. A longitudinal impedance budget of less than $1 \mathrm{Ohm}$ is reasonable for such a ring. Thus longitudinal microwave instability should not be a problem.

* Operated by the Universities Research Association, Inc., under contract with the U.S. Department of Energy. 
Table I: Properties of the $3 \mathrm{TeV}$ low-field ring.

\begin{tabular}{lrr}
\hline \hline & Injection & Extraction \\
\hline Circumference $C(\mathrm{~km})$ & 34.00 & 34.00 \\
Kinetic Energy $(\mathrm{GeV})$ & 150.00 & 3000.00 \\
Gamma $\gamma$ & 160.868 & 3198.37 \\
Revolution frequency $f_{0}(\mathrm{~Hz})$ & 8817.25 & 8817.42 \\
Number of proton per bunch $N$ & $2.70 \times 10^{11}$ & $2.70 \times 10^{11}$ \\
Number of bunches $M$ & 790 & 790 \\
Rf harmonic $h \quad 6020$ & 6020 \\
Rms bunch length $\sigma_{\ell}(\mathrm{m})$ & 0.5000 & 0.5000 \\
$\quad \sigma_{\tau}(\mathrm{ns})$ & 1.6679 & 0.6186 \\
Average current per bunch $I_{b}(\mathrm{amp})$ & $3.814 \times 10^{-4}$ & $3.814 \times 10^{-4}$ \\
Peak current $I_{\mathrm{pk}}(\mathrm{amp})$ & 10.35 & 27.90 \\
Bunch area $A(\mathrm{eV}-\mathrm{s})$ & 1.50 & 1.50 \\
Rms energy spread $\sigma_{E}$ & $3.161 \times 10^{-4}$ & $4.287 \times 10^{-5}$ \\
Normalized $95 \%$ emittance $(\pi$ m) & $1.50 \times 10^{-5}$ & $1.50 \times 10^{-5}$ \\
Betatron tune $\nu_{\beta}$ & $\sim 50$ & $\sim 50$ \\
Bunch area $A(\mathrm{eV}-\mathrm{s})$ & 1.50 & 1.50 \\
Rms energy spread $\sigma_{E}$ & $3.161 \times 10^{-4}$ & $4.287 \times 10^{-5}$ \\
Transition gamma $\gamma_{t}$ & 35.0 & 35.0 \\
Slippage factor $\eta$ & $7.777 \times 10^{-4}$ & $8.162 \times 10^{-4}$ \\
Synchrotron tune $\nu_{s}$ & $2.661 \times 10^{-3}$ & $1.021 \times 10^{-3}$ \\
Rf Voltage $V_{\mathrm{rf}}(\mathrm{MV})$ & 1.434 & 4.000 \\
Beam pipe radius $b(\mathrm{~cm})$ & 0.90 & 0.90 \\
\hline \hline
\end{tabular}


The limit for transverse longitudinal microwave instability is

$$
\left|Z_{1}^{\perp}\right|=\frac{4 \sqrt{2 \pi}|\eta| E \sigma_{E} n_{r}}{\langle\beta\rangle I_{\mathrm{pk}}}
$$

where the average betatron function $\langle\beta\rangle$ is taken as $R / \nu_{\beta}$ and $n_{r}=f_{r} / f_{0}$ is the revolution harmonic of the driving broad-band impedance, $f_{0}=\omega_{0} /(2 \pi)$ being the revolution frequency. We obtain the limits $\left|Z_{1}^{\perp}\right|=22.6$ and $63.9 \mathrm{MOhm} / \mathrm{m}$. One source of impedance is the resistive wall. According to Eq. (3.2) below, the resistive wall of the beam pipe contributes, respectively, $\left|Z_{1}^{\perp}\right|=13.2$ and $8.06 \mathrm{MOhm} / \mathrm{m}$ at injection and extraction for the frequency $f_{r}=\sigma_{\tau}^{-1}$. However, with all the the bellows shielded, it should not be difficult to maintain a transverse impedance budget for this below these stability limits. Thus, transverse microwave instability should not be a problem.

\section{COUPLED-BUNCH INSTABILITIES}

Coupled-bunch instabilities are driven by narrow resonances, mostly from the higher-order modes of the rf cavities. Without any knowledge of these cavities, it will be hard to make any estimation of the instabilities. However, there is a transverse coupled-bunch instability driven by the resistivity of the beam-pipe wall, which can be studied easily.

The beam pipe is of inner radius $b=0.90 \mathrm{~cm}$. It is made of one inner layer of pure aluminum having $1 \mathrm{~mm}$ thickness with resistivity $\rho=2.65 \times 10^{-8} \mathrm{Ohm}-\mathrm{m}$, and an outer layer of a harder alloy having slightly higher resistivity. The skin depth at one revolution harmonic is

$$
\delta_{1}=\sqrt{\frac{\rho c}{Z_{0} \omega_{0}}}=0.873 \mathrm{~mm},
$$

where $c$ is the velocity of light and $Z_{0}$ is the impedance of free space. As will be shown below, if we assume a residual betatron tune of $\left[\nu_{\beta}\right]=0.4$, the lowest tune-line that causes an instability is at the frequency of $f_{\min }=-0.6 f_{0}=-5290 \mathrm{~Hz}$. There, the skin depth will be $1.13 \mathrm{~mm}$, which is roughly the thickness of the aluminum layer of the beam pipe. Thus, we can assume that no electromagnetic fields will leak out from the beam pipe. 
The resistive-wall impedance can be written as

$$
\left(Z_{1}^{\perp}\right)_{\text {wall }}(\omega)=[1-i \operatorname{sgn}(\omega)] \frac{R \delta_{1}}{b^{3}} \sqrt{\frac{\omega_{0}}{|\omega|}} .
$$

This formula is correct when (1) the skin depth is less than the wall thickness, which we have demonstrated to be roughly true, (2)

$$
\sqrt{\omega} \gg \frac{1}{b} \sqrt{\frac{2 \rho c}{Z_{0}}},
$$

or frequency $f \gg 82.9 \mathrm{~Hz}$, which is very well satisfied, and (3)

$$
\omega^{3 / 2} \ll \frac{2 c}{b} \sqrt{\frac{c Z_{0}}{2 \rho}}
$$

or $f \ll 3.5 \times 10^{12} \mathrm{~Hz}$, which is very much larger than the bunch frequency and even the cutoff frequency of the beam pipe. By the way, Eq. (3.3) can also be written as pipe radius very much larger than skin depth.

There are $M=790$ bunches. If they are situated symmetrically in the accelerator ring, there will be $M$ transverse coupled-bunch modes driven by the resistive wall. The growth rate of the $s$ th mode is given by

$$
\tau_{s}^{-1}=-\frac{e c M I_{b}}{4 \pi E \nu} \sum_{k} \operatorname{Re} Z_{1}^{\perp}\left[\left(M k+s+\nu_{\beta}\right) \omega_{0}\right] F,
$$

where the form factor $F$ is close to unity for low frequencies. Since there are $M$ bunches, for each mode the betatron lines are separated by $M$ revolution harmonics. According to Eq. (3.2), The transverse impedance due to the resistive wall falls off as $|\omega|^{-1 / 2}$. Therefore, the most dangerous mode is the one where one betatron line has a negative frequency closest to zero. If we assume the residual betatron tune to be $\left[\nu_{\beta} \mid=0.4\right.$, that line has the frequency of $f_{\min }=-0.6 f_{0}=-5290 \mathrm{~Hz}$. Retaining only that term in the summation in Eq. (3.5), the most dangerous growth rates are, respectively, 3287 and $165.3 \mathrm{~s}^{-1}$ at injection and extraction. The corresponding growth times are 2.68 and 53.3 turns.

Let us investigate whether the fast growth rates can be lowered by running the machine at a positive chromaticity. This amounts to shifting the bunch spectrum 
towards the positive-frequency side, so that the $n=-0.6$ betatron line only overlaps with the tail of the bunch spectrum. However, the bunch rms frequency spread is $\sigma_{f}=\left(2 \pi \sigma_{\tau}\right)^{-1}=95.4$ and $257.3 \mathrm{MHz}$ for the two energies. Therefore, to have a significant effect, the chromaticities required will be roughly $\xi=\sigma_{f} / f_{0}=1 \times 10^{4}$ and $2.9 \times 10^{4}$ units, for injection and extraction. This is, of course, not practical at all. The main reason is the large size of the ring resulting in too low a revolution frequency $f_{0}$.

Another way to reduce the growth rates is to install octupoles so that there will be an amplitude dependent tune spread. We want each bunch to have so much tune spread that coherency will be lost during the growth time. Roughly the required tune spreads will be $\sim 0.37$ and $\sim 0.018$ for the two energies. Obviously, the tune spread at injection is too large to be acceptable. The last resort is a fast active damper.

\section{MODE COUPLING INSTABILITIES}

For impedances with wavelengths longer than the bunch length, bunch instabilities occur when two stable modes collide. Longitudinal mode-coupling instability will be self-stabilized by the lengthening of the bunch, and is therefore not as important. Transverse mode-coupling instability will lead to beam breakup. Here, we concentrate on the transverse modes. When mode 0 shifts by $\Delta \nu_{s} \sim-\nu_{s}$, it collides with mode -1 and an instability occurs. The threshold is given by

$$
\Delta \nu_{s}=-\frac{i e I_{b}\langle\beta\rangle \omega_{0}}{4 \pi E} \int_{-\infty}^{\infty} Z_{1}^{\perp}(\omega) e^{-\omega^{2} \sigma_{\tau}^{2}} d \omega \sim-\nu_{s}
$$

Due to the symmetry properties of $Z_{1}^{\perp}$, it is only the reactive part which contributes to this instability. If we define an effective inductive transverse impedance

$$
\left(Z_{1}^{\perp}\right)_{\mathrm{eff}}=\frac{\int_{-\infty}^{\infty}-\operatorname{Im} Z_{1}^{\perp}(\omega) e^{-\omega^{2} \sigma_{\tau}^{2}} d \omega}{\int_{-\infty}^{\infty} e^{-\omega^{2} \sigma_{\tau}^{2}} d \omega}
$$

then instability will not occur if

$$
\left(Z_{1}^{\perp}\right)_{\mathrm{eff}} \lesssim \frac{4 \sqrt{\pi} \nu_{s} E \sigma_{\tau} \omega_{0}}{e I_{b}\langle\beta\rangle}
$$


These limits are, respectively, 6.37 and $18.03 \mathrm{MOhms} / \mathrm{m}$ at injection and extraction. For a broad-band impedance that resonates at a frequency much higher than the bunch spectrum, the integral can be approximated and we obtain the threshold $\left.Z_{1}^{\perp}\right|_{\text {eff }}=-\operatorname{Im} Z_{1}^{\perp}(0)$. For the resistive wall, using the transverse impedance given by Eq. (3.2), we obtain

$$
\left(Z_{1}^{\perp}\right)_{\text {eff }}=-\mathcal{I} m\left(Z_{1}^{\perp}\right)_{\text {wall }}\left(\omega_{0}\right) \Gamma\left(\frac{1}{4}\right) \sqrt{\frac{\omega_{0} \sigma_{\tau}}{\pi}}= \begin{cases}48.0 \mathrm{MOhm} / \mathrm{m} & \text { Injection } \\ 29.2 \mathrm{MOhm} / \mathrm{m} & \text { Extraction }\end{cases}
$$

We see that these values exceed the stability limits. 